\title{
Validation of Friedewald, Martin/Hopkins and Sampson Equations in the Low-Density Lipoprotein Cholesterol Estimation with Different Assays
}

Gözde Ertürk Zararsız ${ }^{1,2}$, Serkan Bolat ${ }^{3}$, Ahu Cephe $^{4 \bullet}$, Necla Kochan $^{5 \bullet}$, Serra İlayda Yerlitaş $^{1,2}$, Halef Okan Doğan ${ }^{3}$, Gökmen Zararsız ${ }^{1,2^{*}}$

1 Department of Biostatistics, Erciyes University School of Medicine, Kayseri, Turkey 2 Drug Application and Research Center (ERFARMA), Erciyes University, Kayseri, Turkey

3 Department of Biochemistry, Sivas Cumhuriyet University School of Medicine, Sivas, Turkey

4 Institutional Data Management and Analytics Unit, Erciyes University Rectorate, Kayseri, Turkey

$\mathbf{5}$ İzmir Biomedicine and Genome Center (IBG), İzmir, Turkey

DThese authors contributed equally to this work.

* gokmenzararsiz@erciyes.edu.tr

\begin{abstract}
Background

Low-density lipoprotein cholesterol (LDL-C) is an important biomarker for determining cardiovascular risk and regulating lipid lowering therapy. Therefore, the accurate estimation of LDL-C concentration is essential in cardiovascular disease diagnosis and prognosis. Sampson recently proposed a new formula for the estimation of LDL-C. However, little is known regarding the validation of this formula.

\section{Objectives}

This study aimed to validate this new formula with other well-known formulas in Turkish population, composed of adults.

\section{Methods}

A total of 88,943 participants above 18 years old at Sivas Cumhuriyet University Hospital (Sivas, Turkey) were included to this study. LDL-C was directly measured by homogeneous assays, i.e., Roche, Beckman and Siemens and estimated by Friedewald's, Martin/Hopkins', extended Martin/Hopkins' and Sampson's formulas. The accuracies between the estimations obtained by the formulas and the direct measurements were evaluated both in general and separately for the LDL-C, TG and non-HDL-C sublevels. Linear regression analysis was applied and residual error plots were generated between each estimation and direct measurement method. Coefficient of determination $\left(R^{2}\right)$ and mean absolute deviations were also calculated.

\section{Results}

The results showed that the extended Martin/Hopkins approach was the overall most accurate approach for LDL-C estimation. The results also showed that the highest concordances were obtained between the direct assays with the extended Martin/Hopkins formula calculated with the median statistics obtained from our own population. On the other hand, it was observed that the results of the methods may
\end{abstract}


differ in different assays. Although the best performance was obtained by the Sampson formula in patients with "low LDL-C level and hypertriglyceridemia", the extended Martin/Hopkins approach, calculated from the median statistics of our population, gave the most accurate results in patients with "low LDL-C level or hypertriglyceridemia". Conclusions

In conclusion, in patients with a low LDL-C level or hypertriglyceridemia, the extended Martin/Hopkins method; in patients with a low LDL-C level and hypertriglyceridemia, the Sampson method may be preferred. The validity of the Martin Hopkins' and Sampson's formulas has to be further investigated in different populations.

\section{Introduction}

Cardiovascular diseases (CVDs) are the leading causes of death worldwide which accounts for $32 \%$ of all global deaths in 2019 1. There are many different risk factors that increase the likelihood of developing CVD such as smoking, unhealthy diet, obesity, physical inactivity and excessive use of alcohol consumption. However, elevated low-density lipoprotein cholesterol (LDL-C) concentration is the major risk factor associated with an increased risk of CVD mortality. Clinical studies have shown that there exists a strong and positive correlation between LDL-C concentration and the development and progression of CVD [2,3]. Hence, LDL-C is a major determinant which is used as a target measure in clinical practice guidelines and to investigate appropriate treatment strategies.

The gold standard for measuring LDL-C level is $\beta$-quantification which combines ultracentrifugation and precipitation with poly-anions in order to separate lipoprotein particles [4]. However, $\beta$-quantification, is not convenient for routine use since it is expensive, time consuming and requires a large number of sample batches and other instruments $2,3,5,6$. Therefore, the use of this measuring method is limited to a few specialized laboratories 7 . In 1972, a new method called LDL-C estimation by Friedewald formula was introduced and it has become a new standard in clinical practice guidelines worldwide due to its advantages such as convenient, cost-effective and time-saving compared to the direct method, LDL-C with ultracentrifugation followed by $\beta$-quantification 2 . Although it has been widely adopted in clinical practice there are some limitations to this method. Firstly, division of TG directly by a fixed factor of 5, a fixed ratio of TG:VLDL-C, does not provide an accurate estimate for VLDL-C. Secondly, the Friedewald formula requires fasting serum to accurately estimate LDL-C since chylomicronemia in a non-fasting situation leads to the overestimation of VLDL-C [8]. It is also inappropriate to use Friedewald equation in the presence of high TG concentration $(\mathrm{TG} \geq 400)$ and type III hyperlipidemia. Thirdly, the LDL-C levels below $70 \mathrm{mg} / \mathrm{dL}$ and TG $\geq 150$ underestimates LDL-C levels and this may result in undertreatment of the patients 9 .

To overcome the aforementioned limitations, Martin et al. proposed a novel equation to accurately estimate LDL-C levels. This new equation is called Martin/Hopkins formula which uses an adjustable factor for TG:VLDL-C ratio based on TG and non-high-density lipoprotein cholesterol (non-HDL-C) levels 10]. This formula was validated using a large sample of lipid profiles and it was shown in their study that the Martin/Hopkins formula provides more accurate LDL-C estimates compared to Friedewald formula, especially in patients with LDL-C $<70 \mathrm{mg} / \mathrm{dL}$ and those with elevated TG levels [10]. However, despite very promising results, this method did not reach widespread use. This may be due to the need for further validation with various populations. Since then, many studies have been conducted to demonstrate the validity 
Since Friedewald and Martin/Hopkins formulas are developed and validated for patients with $\mathrm{TG}<400 \mathrm{mg} / \mathrm{dL}$, clinical laboratories generally do not report LDL-C levels for hypertriglyceridemia patients whose TG levels are above $399 \mathrm{mg} / \mathrm{dL}$. Chylomicrons accumulate at high TG levels and may change the association between TG and VLDL-C. Therefore, Friedewald formula causes larger errors in LDL-C estimate. On the other hand, Martin/Hopkins formula includes an adjustable factor parameter for splitting TG levels into categories, however it has never been validated for patients with TG levels above $399 \mathrm{mg} / \mathrm{dL}$ 10,12,16. Therefore, clinical laboratories perform direct chemical assays in order to measure the LDL-C levels at higher TG levels, but these direct assays lack standardization, are time consuming and costly [17]. In 2020, in order to accurately estimate LDL-C levels for patients with TG levels up to $800 \mathrm{mg} / \mathrm{dL}$, Sampson et al. derived a new novel equation (hereafter referred to as the Sampson formula) which uses $\beta$-quantification results obtained out of a population with a high frequency of hypertriglyceridemia 18 . The authors claimed that the new formula not only enables clinicians to report LDL-C levels for patients with hypertriglyceridemia (TG level $\leq 800 \mathrm{mg} / \mathrm{dL}$ ), but also estimates LDL-C levels for patients with normopolidemia and/or low level of LDL-C the same as or more accurate than other existing equations [18. Recently, Sajja et al. performed an extended Martin/Hopkins formula and compared its accuracy with Sampson's formula 19. This extended formula uses strata-specific median ratio of TGs:VLDL-C to estimate LDL-C levels for patients with TG levels between 400 and $799 \mathrm{mg} / \mathrm{dL}$. Their results showed that the extended Martin/Hopkins formula gives a more accurate estimate compared to Friedewald and Sampson formulas at TG levels of 400 to $799 \mathrm{mg} / \mathrm{dL}$ and also performs better at low LDL-C levels [19].

In this study, we used a very large sample size and evaluated the validity of the LDL-C levels estimated by Friedewald, Martin-Hopkins, extended Martin-Hopkins and Sampson formulas with the LDL-C levels measured by some direct assays (i.e., Roche, Beckman and Siemens) using the Turkish population.

\section{Materials and methods}

\section{Study population}

A total of 88,943 samples were included the study. The demographic characteristics of the participants according to analytical platforms were given in Table 1 1 . We reviewed the levels of the HDL-C, LDL-C, triglycerides, and total cholesterol in these samples. Data were obtained by the Sivas Cumhuriyet University Medical Faculty, Department of Biochemistry from March 3, 2011, to December 31, 2019. These parameters are ordered from a wide variety of clinical units in the Medical Faculty. The study was approved by the local ethics committee in accordance with the Declaration of Helsinki (2020-03/05). Since the study was designed as retrospective, no informed consent was obtained from participants. We did not categorize the participants based on fasting status. 


\section{Lipid measurements}

Different systems were used to directly measure HDL-C, LDL-C, triglycerides, and total cholesterol parameters. Detailed measurement procedures were given below according to used systems.

Roche Cobas 8000, c-702 and c-501: Total cholesterol, triglycerides and LDL-C, HDL-C measurements were performed using colorimetric enzymatic reaction.

Siemens Advia 1800: HDL-C levels were determined with Trinder reaction. Triglycerides and LDL-C, HDL-C measurements were performed using colorimetric enzymatic reaction.

Beckman Coulter AU5800: Total cholesterol, triglycerides and LDL-C, HDL-C measurements were performed using colorimetric enzymatic reaction.

\section{Lipid estimations}

Direct measurement of LDL-C (LDL-C $\mathrm{D}_{\mathrm{D}}$ ) was measured directly with one of the Roche, Beckman, or Siemens assays. Friedewald's LDL-C estimation (LDL-C $\mathrm{F}_{\mathrm{F}}$ ) was calculated with the following Friedewald formula 22:

$$
\text { LDL- } \mathrm{C}_{\mathrm{F}}=\mathrm{TC}-\mathrm{HDL}-\mathrm{C}-(\mathrm{TG} / 5)
$$

Sampson's LDL-C estimation (LDL-C $\mathrm{C}_{\mathrm{S}}$ ) was calculated using the least squares formula mentioned by Sampson [18:

$$
\mathrm{LDL}_{\mathrm{S}}=\frac{\mathrm{TC}}{0.948}-\frac{\mathrm{HDL}-\mathrm{C}}{0.971}-\left(\frac{\mathrm{TG}}{8.56}+\frac{\mathrm{TG} \times \text { non-HDL-C }}{2140}-\frac{\mathrm{TG}^{2}}{16100}\right)-9.44
$$

Martin/Hopkins and the extended Martin/Hopkins LDL-C estimations (LDL- $\mathrm{C}_{\mathrm{M}}$ and LDL- $\mathrm{C}_{\mathrm{E}}$, respectively) were calculated using the Martin/Hopkins formula [10]:

$$
\text { LDL-C } \mathrm{M}_{\mathrm{M}}=\mathrm{TC}-\mathrm{HDL}-\mathrm{C}-(\mathrm{TG} / \zeta)
$$

In this formula, $\zeta$ is an adjustable factor and was calculated using the median TG/VLDL-C ratio, which takes into account the sublevels of TG and non-HDL-C levels. For LDL-C $\mathrm{C}_{\mathrm{M}}$ estimation, $\zeta$ was obtained from the strata specific median ratio of TG/VLDL-C in the 180-cell table suggested by 10 . For LDL-C $\mathrm{E}_{\mathrm{E}}, \zeta$ values were estimated using Turkish population data. For this purpose, two-dimensional tables were created and strata specific median ratio of TG/VLDL-C were calculated from different combinations by using the accepted cut-off values for TG and non-HDL-C levels. Tables containing the median ratio of TG/VLDL-C with 30,70, 130, 180, 420 and 780 cells were generated, including 5 and 30 sublevels for TG and 6, 14 and 26 sublevels for non-HDL-C (S Tables). Overall accuracies were calculated for each combination. The most accurate results for the Roche and Siemens assays, and the second most accurate results for Beckman assay were obtained from the coefficients in the 180-cell tables.

Although the Beckman direct assay yielded the most accurate results in 420-cell tables, the overall accuracy was only $0.14 \%$ higher compared to calculations based on 180 -cell tables (Fig 1). For this reason, the 180-cell tables were used to calculate the LDL- $\mathrm{C}_{\mathrm{E}}$ estimations in all of the assays. 


\section{Statistical analysis}

Overall accuracies of LDL-C estimates were calculated for each assay separately. Overall accuracy was defined as the ratio of direct LDL-C $\left(\mathrm{LDL}-\mathrm{C}_{\mathrm{D}}\right)$ in the same category as estimated LDL-C based on estimated LDL-C levels $(<70 \mathrm{mg} / \mathrm{dL}, 70$ to 99 $\mathrm{mg} / \mathrm{dL}, 100$ to $129 \mathrm{mg} / \mathrm{dL}, 130$ to $159 \mathrm{mg} / \mathrm{dL}, 160$ to $189 \mathrm{mg} / \mathrm{dL}$ and $\geq 190 \mathrm{mg} / \mathrm{dL}$ ). In addition, overall accuracies for LDL-C estimates were also calculated for the TG and non-HDL-C sublevels. Ordinary least squares linear regression analyses were conducted to compare the estimated and measured LDL-C values. Residual error plots were also generated from the difference of each LDL-C estimation method and direct LDL-C measurements according to TG levels. All analyses were conducted using R 4.0.4 (www.r-project.org) statistical software.

\section{Results}

\section{Patient characteristics}

Demographic details of all participants are provided in Table 1 1 . In this study, a total of 88,943 profiles were taken, out of this $52 \%$ were females and $48 \%$ were males. The mean age of all participants was $53.04 \pm 17.64$. The median values of LDL-C measured by direct method, TC, TG, and HDL-C levels were $114 \mathrm{mg} / \mathrm{dL}, 181 \mathrm{mg} / \mathrm{dL}, 130 \mathrm{mg} / \mathrm{dL}$, and $42 \mathrm{mg} / \mathrm{dL}$, respectively. The median of nonHDL-C level, and TG/TC ratio were calculated as $136 \mathrm{mg} / \mathrm{dL}$ and $0.74 \mathrm{mg} / \mathrm{dL}$, respectively. The demographic details of participants whose HDL-C, LDL-C, triglycerides and total cholesterol parameters are measured by Roche $(N=39,558)$, Beckman $(N=30,087)$ and Siemens $(N=19,298)$ direct assays are also given in Table 1 , separately.

\section{Overall accuracies of the different equations for LDL-C estimation}

In order to estimate LDL-C levels using Martin/Hopkins' formula, strata specific median ratio of TG/VLDL-C were calculated from different combinations by using the accepted cut-off values for TG and non-HDL-C levels. To this end, two-dimensional tables containing the median ratio of TG/VLDL-C with 30, 70, 130, 180, 420 and 780 cells were generated, including 5 and 30 sublevels for TG and 6, 14 and 26 sublevels for non-HDL-C.

Overall accuracies of LDL-C estimates for each assay are given in Fig 1. It can be seen from the figure that the overall accuracies of the different equations implemented in this study to estimate LDL-C levels for Siemens direct method are lower than those with other two direct assays: Roche and Beckman. It can also be seen that the extended Martin/Hopkins with different number of cells produced the highest accuracies for each assay. Even for tables with 30 cells, the accuracy levels for Roche and Siemens were similar with tables with cells greater than 30. Surprisingly, the most accurate results were obtained when extended Martin/Hopkins formula with 180-cell table was performed for Roche and Beckman direct assays. In Siemens direct assay, although the highest accuracy was obtained for the 420 -cell table, there was a very slight increase in the accuracy $(0.14 \%)$ compared to the 180-cell table. Thus, we focused on 180-cell table for the extended Martin/Hopkins formula for all assays. The 180-cell table, which includes the median statistics for TG/VLDL-C ratio is given in Table 2 and the other combinations are given in $\mathbf{S}$ Tables 
Table 1. Study population characteristics.

\begin{tabular}{|c|c|c|c|c|}
\hline Characteristic & $\begin{array}{l}\text { Overall } \\
(N=88,943)\end{array}$ & $\begin{array}{l}\text { Roche } \\
(N=39,558)\end{array}$ & $\begin{array}{l}\text { Beckman } \\
(N=30,087)\end{array}$ & $\begin{array}{l}\text { Siemens } \\
(N=19,298)\end{array}$ \\
\hline Age (years) & $53.04 \pm 17.64$ & $52.85 \pm 17.71$ & $52.61 \pm 17.69$ & $54.08 \pm 17.39$ \\
\hline \multicolumn{5}{|l|}{ Gender } \\
\hline Female & $46,246(52.0)$ & $21,186(53.6)$ & $15,614(51.9)$ & $9,446(48.9)$ \\
\hline Male & $42,697(48.0)$ & $18,372(46.3)$ & $14,473(48.1)$ & $9,852(51.1)$ \\
\hline \multicolumn{5}{|l|}{ Lipid values } \\
\hline TC (mg/dL) & $181(150-213)$ & $177(147-208)$ & $191(160-226)$ & $173(144-205)$ \\
\hline TG (mg/dL) & $130(92-188)$ & $130(93-188)$ & $128(90-186)$ & $131(92-192)$ \\
\hline HDL-C (mg/dL) & $42(35-51)$ & $42(35-52)$ & $44(37-52)$ & $40(33-49)$ \\
\hline Non-HDL-C $(\mathrm{mg} / \mathrm{dL})$ & $136(108-167)$ & $132(104-162)$ & $146(117-178)$ & $131(104-160)$ \\
\hline TG - TC ratio & $0.74(0.54-1.03)$ & $0.76(0.56-1.04)$ & $0.68(0.50-.96)$ & $0.78(0.56-1.10)$ \\
\hline $\mathrm{LDL} \mathrm{C}_{\mathrm{D}}(\mathrm{mg} / \mathrm{dL})$ & $114(88-141)$ & $112(87-139)$ & $127(102-152)$ & $98(75-123)$ \\
\hline LDL-C $_{F}(\mathrm{mg} / \mathrm{dL})$ & $106(81-133)$ & $102(78-128)$ & $116(91-144)$ & $101(77-126)$ \\
\hline LDL-C $_{S}(\mathrm{mg} / \mathrm{dL})$ & $109(85-136)$ & $105(81-131)$ & $119(94-147)$ & $104(80-129)$ \\
\hline LDL-C $_{M}(\mathrm{mg} / \mathrm{dL})$ & $110(86-137)$ & $106(82-132)$ & $120(95-147)$ & $105(82-131)$ \\
\hline $\operatorname{LDL} C_{E}(\mathrm{mg} / \mathrm{dL})$ & $115(90-141)$ & $113(88-139)$ & $129(104-154)$ & $99(77-123)$ \\
\hline
\end{tabular}

Values are expressed as $N(\%)$, mean \pm SD or median $\left(1^{s t}-3^{\text {rd }}\right.$ quartiles $)$. TC: total cholesterol; TG: triglycerides; HDL-C: high-density lipoprotein cholesterol; LDL-C: low-density lipoprotein cholesterol; LDL-C: low-density lipoprotein cholesterol; LDL-C ${ }_{D}$ : LDL-C measured by direct assay; LDL-C $F$ : LDL-C calculated by Friedewald formula; LDL-C S $_{\text {: LDL-C calculated by Sampson formula; LDL-C }}$ : LDL-C calculated by Martin/Hopkins formula; LDL- $\mathrm{C}_{\mathrm{E}}$ : LDL-C calculated by the extended Martin/Hopkins formula.

Fig 1. Overall accuracies of the different equations for LDL-C estimation. Overall accuracies of different equations for LDL-C estimation for each assay (i.e., Roche, Siemens and Beckman) are given in a clustered bar chart. Each bar indicates the accuracy of estimating LDL-C levels by different formulas given that the LDL-C levels measured by Roche, Siemens and Beckman direct methods, accordingly. 
Table 2. Median statistics for the ratio of triglycerides to very low-density lipoprotein cholesterol by the cross table of non-high-density lipoprotein cholesterol and triglycerides calculated from the Turkish population (calculated for each direct assay method for 180-cell strata).

\begin{tabular}{|c|c|c|c|c|c|c|}
\hline \multirow{2}{*}{$\begin{array}{l}\text { TG Levels } \\
(\mathrm{mg} / \mathrm{dL})\end{array}$} & \multicolumn{6}{|c|}{ Non-HDL-C (mg/dL) } \\
\hline & $<100$ & 100-129 & 130-159 & $160-189$ & $190-219$ & $\geq \mathbf{2 2 0}$ \\
\hline 7-49 & R:6.00 B:6.82 S:2.34 & R:5.22 B:4.04 S:2.10 & R:3.50 B:3.91 S:1.50 & R:4.43 B:2.01 S:1.21 & R:2.11 B:2.07 S:0.62 & R:2.11 B:1.02 S:0.62 \\
\hline $50-56$ & R:7.00 B:8.17 S:3.11 & R:5.60 B:5.00 S:2.47 & R:4.23 B:3.50 S:1.92 & R:3.93 B:2.24 S:2.15 & R:2.23 B:2.83 S:0.98 & R:3.65 B:1.98 S:0.39 \\
\hline $57-61$ & R:7.13 B:9.50 S:3.20 & $\mathbf{R}: 6.67 \mathrm{~B}: 6.10 \mathrm{~S}: 2.73$ & R:4.83 B:4.46 S:2.32 & R:3.69 B:3.63 S:2.63 & R:2.35 B:2.68 S:1.21 & R:2.35 B:3.05 S:0.99 \\
\hline $62-66$ & R:7.22 B:9.88 S:3.37 & R:7.11 B:6.40 S:2.52 & R:5.73 B:4.77 S:2.17 & R:4.74 B:2.71 S:2.46 & R:4.40 B:2.54 S:1.41 & R:12.80 B:1.99 S:1.41 \\
\hline 67-71 & R:7.67 B:9.57 S:3.68 & R:6.41 B:7.05 S:2.84 & R:5.38 B:4.63 S:2.53 & R:5.00 B:3.55 S:2.18 & R:2.63 B:2.65 S:2.92 & R:2.09 B:1.43 S:2.92 \\
\hline $72-75$ & R:7.20 B:9.25 S:3.75 & R:6.82 B:7.40 S:3.13 & R:5.73 B:4.93 S:2.71 & R:5.14 B:4.00 S:2.03 & R:4.55 B:2.64 S:1.77 & $\mathrm{R}: 2.62 \mathrm{~B}: 2.11 \mathrm{~S}: 1.77$ \\
\hline 76-79 & R:7.80 B:9.75 S:3.66 & R:7.05 B:7.70 S:3.14 & R:5.64 B:5.64 S:3.16 & R:5.43 B:3.71 S:2.71 & R:2.97 B:2.75 S:2.35 & $\mathbf{R}: 2.81 \mathbf{B}: 2.60 \mathbf{S}: 0.80$ \\
\hline $80-83$ & R:7.36 B:13.33 S:3.91 & R:6.88 B:7.77 S:3.47 & R:5.86 B:5.19 S:2.89 & R:5.63 B:4.37 S:3.20 & R:4.21 B:2.85 S:1.74 & $\mathbf{R}: 2.65$ B:2.35 S:2.37 \\
\hline $84-87$ & R:7.82 B:10.88 S:4.25 & R:7.82 B:7.17 S:3.41 & R:6.00 B:5.67 S:3.26 & R:5.59 B:3.58 S:2.29 & R:5.12 B:3.19 S:1.49 & R:8.40 B:2.97 S:1.76 \\
\hline $88-92$ & R:8.00 B:12.04 S:4.09 & R:8.18 B:7.42 S:3.83 & R:6.13 B:5.93 S:3.54 & R:5.08 B:4.74 S:2.73 & R:4.05 B:3.37 S:1.97 & R:2.94 B:2.49 S:1.75 \\
\hline 93-96 & R:8.55 B:10.56 S:4.43 & R:7.15 B:8.00 S:3.96 & R:6.52 B:5.88 S:3.43 & R:5.53 B:4.36 S:3.29 & R:6.33 B:3.72 S:3.77 & R:4.89 B:3.47 S:1.08 \\
\hline 97-100 & R:7.54 B:12.38 S:4.25 & R:7.69 B:8.82 S:3.90 & R:6.96 B:6.53 S:3.05 & R:6.57 B:4.81 S:3.05 & R:5.00 B:3.59 S:2.19 & R:6.19 B:2.56 S:1.95 \\
\hline 101-105 & R:7.92 B:11.44 S:4.81 & R:8.00 B:8.58 S:4.13 & R:7.11 B:6.44 S:3.68 & R:6.50 B:5.00 S:2.94 & R:6.71 B:4.21 S:3.76 & R:3.74 B:2.81 S:2.25 \\
\hline 106-110 & R:8.27 B:12.06 S:4.91 & R:7.33 B:9.08 S:4.09 & R:7.64 B:6.78 S:3.53 & R:6.47 B:5.24 S:2.69 & R:5.40 B:3.79 S:2.69 & R:5.00 B:3.03 S:3.72 \\
\hline 111-115 & R:8.14 B:12.33 S:5.14 & R:7.67 B:9.25 S:4.63 & R:7.96 B:7.13 S:3.99 & R:6.74 B:4.87 S:3.35 & R:5.23 B:4.11 S:3.29 & R:4.48 B:3.02 S:3.06 \\
\hline 116-120 & R:7.73 B:12.00 S:5.29 & R:7.87 B:9.19 S:4.33 & R:7.44 B:6.82 S:4.20 & R:7.00 B:5.27 S:3.55 & R:5.65 B:4.46 S:3.53 & R:10.90 B:3.08 S:3.24 \\
\hline 121-126 & R:8.40 B:15.75 S:5.30 & R:8.13 B:10.25 S:4.31 & R:7.35 B:7.69 S:4.25 & R:7.09 B:5.70 S:3.73 & R:6.15 B:4.54 S:2.80 & R:5.59 B:3.97 S:2.41 \\
\hline $127-132$ & R:8.57 B:15.94 S:5.30 & R:8.19 B:9.77 S:4.45 & R:7.53 B:7.94 S:4.21 & R:7.19 B:5.52 S:3.78 & R:6.19 B:4.40 S:3.12 & R:5.33 B:3.49 S:3.11 \\
\hline 133-138 & R:7.75 B:14.78 S:5.04 & R:8.59 B:10.42 S:4.74 & R:8.06 B:7.44 S:4.15 & R:6.90 B:6.11 S:3.79 & R:5.90 B:5.06 S:3.45 & R:6.18 B:3.62 S:2.84 \\
\hline $139-146$ & R:8.26 B:16.06 S:5.33 & R:8.75 B:10.21 S:4.71 & R:7.94 B:8.29 S:4.77 & R:7.28 B:5.88 S:4.25 & R:7.42 B:5.15 S:3.47 & R:5.32 B:3.75 S:2.90 \\
\hline $147-154$ & R:7.84 B:15.92 S:5.52 & R:8.82 B:11.38 S:5.07 & R:8.28 B:8.44 S:4.61 & R:7.45 B:6.67 S:3.94 & R:6.61 B:4.78 S:3.75 & R:5.65 B:3.76 S:3.20 \\
\hline $155-163$ & R:8.00 B:17.44 S:6.00 & R:8.37 B:11,43 S:4.89 & R:7.93 B:9.53 S:4.88 & R:7.80 B:6.87 S:4.30 & R:6.87 B:5.61 S:4.03 & R:5.59 B:4.45 S:3.25 \\
\hline 164-173 & R:7.86 B:15.41 S:5.90 & R:8.38 B:12.69 S:5.44 & R:8.05 B:9.88 S:4.86 & R:7.59 B:7.26 S:4.29 & R:6.58 B:5.39 S:3.98 & R:5.43 B:5.34 S:3.38 \\
\hline 174-185 & R:7.87 B:16.27 S:6.79 & R:8.32 B:12.71 S:5.68 & R:8.76 B:9.89 S:5.00 & R:8.02 B:7.36 S:4.38 & R:7.50 B:6.07 S:3.87 & R:5.76 B:4.14 S:3.69 \\
\hline 186-201 & R:8.64 B:18.09 S:6.19 & R:8.59 B:12.87 S:5.89 & R:8.38 B:10.34 S:5.37 & R:7.72 B:8.78 S:4.75 & R:7.15 B:6.46 S:4.12 & R:5.68 B:5.03 S:3.58 \\
\hline $202-220$ & R:8.15 B:18.07 S:6.11 & R:8.76 B:14.71 S:6.34 & R:8.83 B:11.16 S:5.63 & R:8.20 B:8.46 S:5.09 & R:7.59 B:6.66 S:4.69 & R:6.77 B:5.81 S:4.40 \\
\hline $221-247$ & R:8.81 B:21.27 S:6.94 & R:8.79 B:15.77 S:6.47 & R:8.67 B:11.60 S:5.70 & R:8.23 B:9.04 S:5.00 & R:7.44 B:7.09 S:4.92 & R:7.00 B:5.66 S:4.42 \\
\hline 248-292 & R:8.56 B:20.77 S:7.14 & R:8.66 B:15.78 S:6.49 & R:8.83 B:12.13 S:5.82 & R:8.23 B:10.00 S:5.37 & R:7.85 B:8.29 S:5.02 & R:7.15 B:6.16 S:4.40 \\
\hline 293-399 & R:8.76 B:24.86 S:7.82 & R:8.69 B:19.15 S:7.09 & R:8.68 B:14.77 S:6.48 & R:8.05 B:11.44 S:5.79 & R:7.75 B:9.45 S:5.25 & R:7.15 B:6.83 S:4.56 \\
\hline$\geq 400$ & R:12.16 B:23.56 S:8.83 & R:8.54 B:17.93 S:7.45 & R:8.90 B:15.31 S:7.03 & R:8.26 B:12.99 S:6.51 & R:7.54 B:11.03 S:6.16 & R:4.90 B:8.87 S:4.59 \\
\hline
\end{tabular}




\section{Distribution density of LDL-C concentrations calculated by direct methods and different formulas}

The raincloud plots of measured LDL-C levels by direct methods and LDL-C estimates using the formulas considered in this study are provided by Fig $\mathbf{2}$. The red line in this figure shows the difference between the median of measured LDL-C with Roche, Beckman or Siemens and the median of estimated LDL-C levels. It is evident from the figure that all of the formulas except extended Martin/Hopkins formula underestimated the LDL-C levels when Roche and Beckman assays were used, whereas all formulas except Friedewald's formula overestimated the LDL-C levels when Siemens direct assay was utilized. On the other hand, the distribution pattern for this assay was the most similar, with Martin/Hopkins formula.

Fig 2. Distribution density of LDL-C concentrations calculated by direct methods and different formulas. Box-plots are also represented to compare the LDL-C levels measured by direct method with the LDL-C levels estimated by Friedewald, Sampson, Martin/Hopkins and extended Martin/Hopkins formulas. Red dash line is depicted to see the difference between direct method (Roche, Beckman or Siemens) and the formulas used to measure the LDL-C levels.

\section{Accuracies of the different equations for LDL-C estimation by LDL-C strata}

Accuracies of the different equations for LDL-C estimation by different LDL-C sublevels are given in Fig 3 . For Roche direct assay, even though Friedewald and the extended Martin/Hopkins equations gave the most accurate results when LDL-C is less than 70 $\mathrm{mg} / \mathrm{dL}$, the performance of the Martin/Hopkins equation surpasses the performance of the Friedewald and the extended Martin/Hopkins equations, as well as the performances of the other equations when LDL-C level is between $70 \mathrm{mg} / \mathrm{dL}$ and 99 $\mathrm{mg} / \mathrm{dL}$. The extended Martin/Hopkins method outperformed the other equations when LDL-C level is higher than $99 \mathrm{mg} / \mathrm{dL}$. The Martin/Hopkins method provided the most accurate results for Beckman direct assay when LDL-C measured below $70 \mathrm{mg} / \mathrm{dL}$.

However, the performance of the extended Martin/Hopkins was higher than these three methods (i.e., Friedewald, Sampson and Martin/Hopkins) when LDL-C level is above 70 $\mathrm{mg} / \mathrm{dL}$. For Siemens direct assay, the overall accuracies are lower than the other assays, but extended Martin/Hopkins gave the most accurate results for LDL-C levels measured up to $129 \mathrm{mg} / \mathrm{dL}$ and Martin/Hopkins for LDL-C levels beyond $129 \mathrm{mg} / \mathrm{dL}$.

Fig 3. Accuracies of the different equations for LDL-C estimation by LDL-C strata. Accuracies of different equations for LDL-C estimation by LDL-C groups assuming different direct measures (i.e., Roche, Beckman and Siemens) are given in a clustered bar chart. Each bar indicates the accuracy of estimating LDL-C levels by different formulas for each group of LDL-C levels given that the LDL-C levels measured by Roche, Beckman and Siemens direct methods, accordingly. 


\section{Accuracies of the different equations for LDL-C estimation by triglycerides strata}

Overall accuracies for LDL-C estimates by five different triglycerides sublevels $(<100$ $\mathrm{mg} / \mathrm{dL}, 100$ to $149 \mathrm{mg} / \mathrm{dL}, 150$ to $199 \mathrm{mg} / \mathrm{dL}, 200$ to $329 \mathrm{mg} / \mathrm{dL}$ and $\geq 400 \mathrm{mg} / \mathrm{dL}$ ) were given in Fig 4. The results showed that the extended Martin/Hopkins gave the highest accuracy for each assay and for any TG sublevels. However, while the TG levels increased, the accuracies of the equations decreased for any direct assays. The accuracy of the equations used for LDL-C estimation given five different TG sublevels with six different LDL-C strata was also calculated (S1 Fig, S6 Fig).

Fig 4. Accuracies of the different equations for LDL-C estimation by triglycerides strata. Accuracies of different equations for LDL-C estimation by triglycerides groups for different direct measures (i.e., Roche, Beckman and Siemens) are given in a clustered bar chart. Each bar indicates the accuracy of estimating LDL-C levels by different formulas for each group of triglycerides concentration given that the LDL-C levels measured by Roche, Beckman and Siemens direct methods, accordingly.

When the performances of the methods were evaluated according to the TG changes in LDL-C sublevels, we found that the results differed from assay to assay $\mathbf{S 1}$ Fig, S6 Fig. In patients with LDL-C $<70 \mathrm{mg} / \mathrm{dL}$, we observed that the accuracies of Friedewald, Sampson and Martin/Hopkins formulas for TG $<150 \mathrm{mg} / \mathrm{dL}$ for Roche and Beckman assays were quite similar and provided the highest results compared to the extended Martin/Hopkins formula. We found that the performance of Martin/Hopkins and Friedewald methods decreased significantly as TG increased for the Roche assay, while this decrease was comparatively less for the Sampson formula. For TG $>=400$ $\mathrm{mg} / \mathrm{dL}$, Sampson equation gave the most accurate and the extended Martin/Hopkins equation gave the second most accurate results. We observed that the results of the Martin/Hopkins method were most accurate as TG increases for the Beckman assay. For this assay, we could not calculate the performances of the methods because the number of observations was very low in the LDL-C $<70 \mathrm{mg} / \mathrm{dL}$ and TG $\geq 400 \mathrm{mg} / \mathrm{dL}$ scenarios. For Siemens assay, while the performance of the extended Martin/Hopkins method was highest in the TG $<150 \mathrm{mg} / \mathrm{dL}$ scenario, we found a significant increase in the performance of the Friedewald method when the TG levels were between 150 and $400 \mathrm{mg} / \mathrm{dL}$. For TG $\geq 400$, Sampson and the extended Martin/Hopkins equations provided the best results with very similar accuracies S1 Fig For LDL-C between 70 to $99 \mathrm{mg} / \mathrm{dL}$, in most scenario, the accuracy of Martin/Hopkins equation for Roche assay and the extended Martin/Hopkins for the other assays were observed to be the highest S2 Fig. Sampson equation provided the most accurate results for Siemens assay for TG $\geq 400 \mathrm{mg} / \mathrm{dL}$. It is obvious from S3 Fig that extended Martin/Hopkins method performed better than other methods for each assay with any TG sublevels when LDL-C levels were between 100 and $129 \mathrm{mg} / \mathrm{dL}$. The same pattern can be seen for the LDL-C sublevels (130 to $159 \mathrm{mg} / \mathrm{dL}$ and 160 to $189 \mathrm{mg} / \mathrm{dL}$ ) for Roche and Beckman direct assays (S4 Fig, S5 Fig). When LDL-C is measured above $189 \mathrm{mg} / \mathrm{dL}$ by Roche direct assay, while the most accurate estimate was obtained by the Sampson method for TG $<100 \mathrm{mg} / \mathrm{dL}$, the extended Martin Hopkins formula yielded more accurate LDL-C estimates for TG $\geq 100 \mathrm{mg} / \mathrm{dL}$ compared to other formulas $\mathbf{S 6 ~ F i g}$. When LDL-C is measured above $189 \mathrm{mg} / \mathrm{dL}$ by Beckman direct assay, the Sampson formula performed better than other formulas in the first three TG sublevels whereas the extended Martin/Hopkins indicated the highest accuracy of all methods applied in this study $\mathbf{S 6}$ Fig. When LDL-C is measured above $189 \mathrm{mg} / \mathrm{dL}$ by Siemens direct assay, the extended 

patients with low LDL-C and/or hypertriglyceridemia

LDL-C estimation equations are not well validated for patients with low LDL-C (i.e., LDL-C $<70 \mathrm{mg} / \mathrm{dL}$ ) and/or higher TG levels (i.e., TG $\geq 400 \mathrm{mg} / \mathrm{dL}$ ). For this reason, a special attention is given to the comparison of equations for LDL-C $<70 \mathrm{mg} / \mathrm{dL}$ and/or TG $\geq 400 \mathrm{mg} / \mathrm{dL}$. As seen from Table 3 the extended Martin/Hopkins formula for the Roche and Siemens assays, and Martin/Hopkins formula for Beckman assay gave the most accurate results for patients with low LDL-C levels (Table 3 , Fig 3). For patients with higher TG levels, the extended Martin/Hopkins formula provided the most accurate results (Table 3, Fig 4. When the results were examined for patients with low LDL-C levels and higher TG levels, Sampson formula provided the most accurate results for Roche assay. For Siemens assay, both Sampson and the extended Martin/Hopkins formula gave the same and most accurate results (Table 3 , (S1 Fig.

Table 3. The accuracies of LDL-C estimation equations in patients with low LDL-C and/or hypertriglyceridemia.

\begin{tabular}{|l|l|l|l|l|}
\hline Patient group / Direct assay & Friedewald & Sampson & Martin/Hopkins & $\begin{array}{l}\text { Extended } \\
\text { Martin/Hopkins }\end{array}$ \\
\hline Low LDL-C level & & & & \\
\hline Roche & 0.817 & 0.797 & 0.726 & $\mathbf{0 . 8 1 9}$ \\
\hline Beckman & 0.784 & 0.807 & $\mathbf{0 . 8 2 5}$ & 0.723 \\
\hline Siemens & 0.596 & 0.576 & 0.537 & $\mathbf{0 . 6 3 3}$ \\
\hline Hypertriglyceridemia & & & & \\
\hline Roche & 0.269 & 0.378 & 0.589 & $\mathbf{0 . 6 0 2}$ \\
\hline Beckman & 0.078 & 0.378 & 0.389 & $\mathbf{0 . 6 8 3}$ \\
\hline Siemens & 0.394 & 0.501 & 0.410 & $\mathbf{0 . 5 2 2}$ \\
\hline $\begin{array}{l}\text { Low LDLC level and } \\
\text { hypertriglyceridemia }\end{array}$ & & & & \\
\hline Roche & 0.318 & $\mathbf{0 . 5 8 8}$ & 0.306 & 0.400 \\
\hline Beckman & $\mathrm{NC}$ & $\mathrm{NC}$ & $\mathrm{NC}$ & $\mathrm{NC}$ \\
\hline Siemens & 0.309 & $\mathbf{0 . 5 4 5}$ & 0.164 & $\mathbf{0 . 5 4 5}$ \\
\hline
\end{tabular}

NC: Not computed due to the very low sample size. The most accurate results are shown in bold.

\section{Accuracies of the different equations for LDL-C estimation by non-HDL-C strata}

Overall accuracies for LDL-C estimates by six different non-HDL-C sublevels $(<100$ $\mathrm{mg} / \mathrm{dL}, 100$ to $129 \mathrm{mg} / \mathrm{dL}, 130$ to $159 \mathrm{mg} / \mathrm{dL}, 160$ to $189 \mathrm{mg} / \mathrm{dL}, 190$ to $219 \mathrm{mg} / \mathrm{dL}$ and $\geq 220 \mathrm{mg} / \mathrm{dL}$ ) were given in Fig $\mathbf{5}$. The results showed that extended

Martin/Hopkins gave the highest accuracy for each assay and for almost all non-HDL-C sublevels. Additionally, for Siemens direct assay, while the non-HDL-C levels increased, the accuracy of the methods decreased. The accuracy of the methods used for LDL-C estimation given six different non-HDL-C sublevels with six different LDL-C strata was also evaluated (S7 Fig, S12 Fig). 
Fig 5. Accuracies of the different equations for LDL-C estimation by non-HDL-C strata. Accuracies of different equations for LDL-C estimation by non-HDL-C groups assuming different direct measures (i.e., Roche, Beckman and Siemens) are given in a clustered bar chart. Each bar indicates the accuracy of estimating LDL-C levels by different formulas for each group of non-HDL-C concentration given that the LDL-C levels measured by Roche, Beckman and Siemens direct methods, accordingly.

\section{Regression analysis between LDL-C levels estimated by formulas and directly measured LDL-C levels}

The linear regression analyses demonstrated that estimated LDL-C levels by extended Martin/Hopkins formula indicate better correlation with Roche and Beckman assays with an $\mathrm{R}$ square of 0.91 (Fig $\mathbf{6}$ ). The highest $\mathrm{R}$ square statistic was obtained with the Martin/Hopkins and the extended Martin/Hopkins methods for Siemens direct measurement method. It is obvious to see that Martin/Hopkins and extended Martin/Hopkins show a better association with any direct methods overall.

Fig 6. Regression analysis between LDL-C levels estimated by formulas and directly measured LDL-C levels. Correlations of estimated LDL-C levels by Friedewald, Sampson, Martin/Hopkins and extended Martin/Hopkins formulas with LDL-C levels directly measured by Roche, Beckman and Siemens.

\section{Residual error plots for LDL-C by different formulas with respect to different direct assay methods}

The residual error plots show how the bias between LDL-C estimations calculated by the equations and direct measurements varies according to triglyceride levels (Fig 7$)$. It is seen that the Friedewald formula underestimated the LDL-C levels as the TG level increased in all assays. Similar results were found in the Sampson formula for the Roche and Beckman assays, and in the Martin/Hopkins formula for the Beckman assay. It was observed that the bias in the Siemens assay according to the TG levels was less compared to the other assays. In the Roche and Siemens formulas, the bias for the Martin/Hopkins formula was found to be constant according to the change in TG levels. For the Extended Martin/Hopkins formula, it was determined that the bias in all assays did not change according to TG levels and was close to zero. The lowest mean absolute deviation statistics were obtained with the extended Martin/Hopkins formula for all assays.

Fig 7. Residual error plots for LDL-C by different formulas concerning to different direct assay methods. While the values on x-axis show TG levels, the values on y-axis shows the difference between estimated LDL-C (by Friedewald, Sampson, Martin/Hopkins or extended Martin/Hopkins) and direct LDL-C levels (calculated by Roche, Beckman or Siemens). The mean absolute deviation (MAD) for each possible case is also given in each panel for each dataset. 


\section{Discussion}

The Martin/Hopkins formula has been proposed as a replacement for the Friedewald formula and its validity has been proven in many populations. However, it has been reported that this formula does not have enough accuracy in patients with hypertriglyceridemia. Today, the most up-to-date formula for estimating LDL-C level has been proposed by Sampson et al. [18]. Recent studies have investigated the validity of the Sampson formula in different populations and evaluated whether it produces more accurate results compared to the Martin/Hopkins formula. Song et al. showed that the Martin/Hopkins method gave better results in the East Asian population [20]. Most of the studies stated that the Sampson formula gave the most promising results compared to other formulas 21 24. In one study, it was stated that both Sampson and Martin/Hopkins methods provide the most accurate results 25.

Our study contributes to the literature by validating and comparing Sampson's formula with the Friedewald and Martin/Hopkins equations in different direct assays for estimation of LDL-C. When we evaluated the general accuracy of the methods in our study, we observed that the Martin/Hopkins method gave slightly more accurate results for the Roche and Siemens direct assays, while the methods produced similar results for the Beckman direct assay. However, when we used the median statistics obtained from our own population, we observed that the extended Martin/Hopkins approach produced much more accurate results in all assays compared to the Friedewald, Martin/Hopkins and Sampson methods. Even with the median coefficients obtained from the 30 and 70-cell tables, we observed a significant increase in accuracy compared to the other methods. We observed that the accuracy rates were very similar in tables with cells 70 , 130, 180 and 420 where the median statistics were calculated.

Although we determined that the extended Martin/Hopkins method was the most accurate method for all assays, as a new finding, we found that the results were variable in different assays. For the Beckman assay, the extended Martin/Hopkins method gave the lowest accuracy at low values of LDL-C, while it became the most accurate as the LDL-C level increased. The most accurate results were obtained with the extended Martin/Hopkins method in many cases for the Roche assay. For the Siemens assay, the results were vice versa. In this assay, the performance of the extended Martin/Hopkins method was found to be the best when the LDL-C level was below $130 \mathrm{mg} / \mathrm{dL}$, and the lowest when the LDL-C level was above $130 \mathrm{mg} / \mathrm{dL}$. This low performance may be sourced from the low sample size in these scenarios. LDL-C level was between 130-159 $\mathrm{mg} / \mathrm{dL}$ in $13.4 \%$ of individuals directly measured with the Siemens assay, between $160-189 \mathrm{mg} / \mathrm{dL}$ in $4.4 \%$ of individuals, and $190 \mathrm{mg} / \mathrm{dL}$ and above in $1.5 \%$ of individuals. We observed that the extended Martin/Hopkins method still performed best, although there was variation in the performance of the methods as the triglyceride levels and non-HDL-C levels changed.

Sampson reported that their equation is more accurate than Martin/Hopkins equation for patients with a low LDL-C level and/or hypertriglyceridemia. In our study, when the LDL-C level is below $70 \mathrm{mg} / \mathrm{dL}$, the accuracy of the Martin/Hopkins method for Beckman assay and the extended Martin/Hopkins method for Roche and Siemens assays were found to be the highest. The accuracy of the extended Martin/Hopkins method is highest for all assays in individuals with triglyceride levels above $400 \mathrm{mg} / \mathrm{dL}$. The accuracy of the Sampson method for Roche assay was found to be highest in patients with LDL-C lower than $70 \mathrm{mg} / \mathrm{dL}$ and triglyceride level higher than $400 \mathrm{mg} / \mathrm{dL}$. In the same scenario, the accuracy of Sampson and extended Martin/Hopkins methods for Siemens assay were found to be similar and the highest. Therefore, as Sampson et al. 18 stated, our findings for the Roche assay support the statement that the Sampson 
The accurate estimation of LDL-C is so important to identify the patients who have risk for future cardiovascular disease and the success of the treatment in patients who take lipid lowering drugs ( $[26]$ ). We determined the differences between the measured and estimated LDL-C levels in three different platforms. Certain formulas led to under or over estimations compared to LDL-C measurement with direct assays. The direction and the size of the effects were changing according to analytical platforms and different LDL-C strata. Accordingly, laboratories should determine appropriate formula in the estimation of LDL-C according to their analytical platforms.

In the present study, there was a difference in terms of the accuracy between formula in patients with $<70 \mathrm{mg} / \mathrm{dL}$ LDL-C levels. Accurate estimation of the LDL-C levels below $70 \mathrm{mg} / \mathrm{dl}$ is so important for patients who have higher risk for cardiovascular disease to avoid the under-treatment.

\section{Conclusion}

Although the results of the methods in different assays may vary, the extended Martin/Hopkins approach is the method with the highest overall accuracy. When using the Martin/Hopkins formula, researchers must calculate the median statistics from their own population. Also, the extended Martin/Hopkins approach is the best approach for patients with a low LDL-C level or hypertriglyceridemia. In patients with a low LDL-C level and hypertriglyceridemia, the accuracy of the Sampson method was found to be the best method for the Roche assay. Further validation is warranted in different populations.

\section{Supporting information}

S1 Fig. Accuracies of the different equations for LDL-C estimation by triglycerides strata (LDL $<70 \mathrm{mg} / \mathrm{dL})$.

S2 Fig. Accuracies of the different equations for LDL-C estimation by triglycerides strata (LDL between 70 to $99 \mathrm{mg} / \mathrm{dL}$ ).

S3 Fig. Accuracies of the different equations for LDL-C estimation by triglycerides strata (LDL between 100 to $129 \mathrm{mg} / \mathrm{dL}$ ).

S4 Fig. Accuracies of the different equations for LDL-C estimation by triglycerides strata (LDL between 130 to $159 \mathrm{mg} / \mathrm{dL}$ ).

S5 Fig. Accuracies of the different equations for LDL-C estimation by triglycerides strata (LDL between 160 to $189 \mathrm{mg} / \mathrm{dL}$ ). 
S7 Fig. Accuracies of the different equations for LDL-C estimation by nonHDL-C strata (LDL $<70 \mathrm{mg} / \mathrm{dL})$.

S8 Fig. Accuracies of the different equations for LDL-C estimation by nonHDL-C strata (LDL between 70 to $99 \mathrm{mg} / \mathrm{dL}$ ).

S9 Fig. Accuracies of the different equations for LDL-C estimation by nonHDL-C strata (LDL between 100 to $129 \mathrm{mg} / \mathrm{dL}$ ).

S10 Fig. Accuracies of the different equations for LDL-C estimation by nonHDL-C strata (LDL between 130 to $159 \mathrm{mg} / \mathrm{dL}$ ).

S11 Fig. Accuracies of the different equations for LDL-C estimation by nonHDL-C strata (LDL between 160 to $189 \mathrm{mg} / \mathrm{dL}$ ).

S12 Fig. Accuracies of the different equations for LDL-C estimation by nonHDL-C strata (LDL $\geq 190 \mathrm{mg} / \mathrm{dL}$ ).

S Tables. Median statistics for the ratio of triglycerides to very low-density lipoprotein cholesterol by the cross table of non-high-density lipoprotein cholesterol and triglycerides calculated from the Turkish population.

\section{References}

1. World Health Organisation. Cardiovascular diseases (CVDs); 2021. Available from: https://www.who.int/news-room/fact-sheets/detail/ cardiovascular-diseases-(cvds) [cited 2022 January 10].

2. Friedewald W, Levy R, Fredrickson D. Estimation of the concentration of low density lipoproteins cholesterol in plasma without use of the ultracentrifuge. Clin Chem. 1972;18:499-502.

3. Cordova CMM, Schneider CR, Juttel ID, Cordova MM. Comparison of LDL-cholesterol direct measurement with the estimate using the Friedewald formula in a sample of 10,664 patients. Arq Bras Cardiol. 2004;83:482-87.

4. Centers for Disease Control and Prevention Cholesterol Reference Method Laboratory Network (CRMLN). Total Cholesterol Certification Protocol for Manufacturers-Revised; 2004. Available from: https:

//www.cdc.gov/labstandards/pdf/crmln/RevisedTCprotocol0ct04.pdf [cited 2022 January 10].

5. Cordova CMM, Schneider, Cordova MM. A new accurate, simple formula for LDL-cholesterol estimation based on directly measured blood lipids from a large cohort. Ann Clin Biochem. 2004;50(Pt 1):13-19.

6. Türkalp I, Cil Z, Özkazanç D. Analytical performance of a direct assay for LDL-cholesterol: a comparative assessment versus Friedewald's formula. Anadolu Kardiyol Derg. 2005;5(1):13-17. 
medRxiv preprint doi: https://doi.org/10.1101/2022.01.29.22270067; this version posted January 30, 2022. The copyright holder for this preprint (which was not certified by peer review) is the author/funder, who has granted medRxiv a license to display the preprint in perpetuity.

It is made available under a CC-BY 4.0 International license .

7. National cholesterol Education Program Working Group on Lipoprotein Measurement: Recommendations on Lipoprotein Measurement. NIH Publication No 95-3044. 1995:31-34;

8. Chung S. Usefulness of the Martin Method for Estimation of Low-Density Lipoprotein Cholesterol in Coronary Atherosclerosis. Medical Principles and Practice. 2017;27:8 - 14 .

9. Hermans MP, Ahn SA, Rousseau MF. Novel unbiased equations to calculate triglyceride-rich lipoprotein cholesterol from routine non-fasting lipids.

Cardiovasc Diabetol. 2014;13(56).

10. Martin SS, Blaha MJ, Elshazly MB, Toth PP, Kwiterovich PO, Blumenthal RS, et al. Comparison of a Novel Method vs the Friedewald Equation for Estimating Low-Density Lipoprotein Cholesterol Levels From the Standard Lipid Profile. JAMA. 2013;310(19):2061-2068. doi:10.1001/jama.2013.280532.

11. Sathiyakumar V, Park J, Quispe R, Elshazly MB, Michos ED, Banach M, et al. Impact of Novel Low-Density Lipoprotein-Cholesterol Assessment on the Utility of Secondary Non-High-Density Lipoprotein-C and Apolipoprotein B Targets in Selected Worldwide Dyslipidemia Guidelines. Circulation. 2018;138(3):244-254. doi:10.1161/CIRCULATIONAHA.117.032463.

12. Sathiyakumar V, Blumenthal RS, Elshazly MB. New Information on Accuracy of LDL-C Estimation. American College of Cardiology. 2020; p. 1-11.

13. Chaen H, Kinchiku S, Miyata M, Kajiya S, Uenomachi H, Yuasa T, et al. Validity of a Novel Method for Estimation of Low-Density Lipoprotein Cholesterol Levels in Diabetic Patients. Journal of Atherosclerosis and Thrombosis. 2016;23(12):1355-1364. doi:10.5551/jat.35972.

14. Shin D, Bohra C, Kongpakpaisarn K. Novel method versus the Friedewald method for estimating low-density lipoprotein cholesterol in determination of the eligibility for statin treatment for primary prevention in the United States. Medicine. 2018;97.

15. Grundy SM, Stone NJ, Bailey AL, Beam C, Birtcher KK, Blumenthal RS, et al. 2018

AHA/ACC/AACVPR/AAPA/ABC/ACPM/ADA/AGS/APhA/ASPC/NLA/PCNA Guideline on the Management of Blood Cholesterol: A Report of the American College of Cardiology/American Heart Association Task Force on Clinical Practice Guidelines. Circulation. 2019;139(25):e1082-e1143. doi:10.1161/CIR.0000000000000625.

16. Martin SS, Giugliano RP, Murphy SA, Wasserman SM, Stein EA, Češka R, et al. Comparison of Low-Density Lipoprotein Cholesterol Assessment by Martin/Hopkins Estimation, Friedewald Estimation, and Preparative Ultracentrifugation: Insights From the FOURIER Trial. JAMA Cardiology. 2018;3(8):749-753. doi:10.1001/jamacardio.2018.1533.

17. Brownstein AJ, Martin SS. More accurate LDL-C calculation: Externally validated, guideline endorsed. Clinica Chimica Acta. 2020;506:149-153. doi:https://doi.org/10.1016/j.cca.2020.03.030. 
18. Sampson M, Ling C, Sun Q, Harb R, Ashmaig M, Warnick R, et al. A New Equation for Calculation of Low-Density Lipoprotein Cholesterol in Patients With Normolipidemia and/or Hypertriglyceridemia. JAMA Cardiology. 2020;5(5):540-548. doi:10.1001/jamacardio.2020.0013.

19. Sajja A, Park J, Sathiyakumar V, Varghese B, Pallazola VA, Marvel FA, et al. Comparison of Methods to Estimate Low-Density Lipoprotein Cholesterol in Patients With High Triglyceride Levels. JAMA Network Open. 2021;4(10):e2128817-e2128817. doi:10.1001/jamanetworkopen.2021.28817.

20. Song Y, Lee HS, Baik SJ, Jeon S, Han D, Choi SY, et al. Comparison of the effectiveness of Martin's equation, Friedewald's equation, and a Novel equation in low-density lipoprotein cholesterol estimation. Sci Rep. 2021;11(1):13545.

21. Piani F, Cicero AFG, Ventura F, Dormi A, Fogacci F, Patrono D, et al. Evaluation of twelve formulas for LDL-C estimation in a large, blinded, random Italian population. Int J Cardiol. 2021;330:221-227.

22. Piani F, Cicero AFG, Borghi C, D'Addato S, BLIP Study group. Is the 2020 Sampson equation the best formula for LDL-C estimation? Eur J Intern Med. 2021;83:99-101.

23. Vargas-Vázquez A, Bello-Chavolla OY, Antonio-Villa NE, Mehta R, Cruz-Bautista I, Aguilar-Salinas CA. Comparative assessment of LDL-C and VLDL-C estimation in familial combined hyperlipidemia using Sampson's, Martin's and Friedewald's equations. Lipids Health Dis. 2021;20(1):46.

24. Cicero AFG, Fogacci F, Patrono D, Mancini R, Ramazzotti E, Borghi C, et al. Application of the Sampson equation to estimate LDL-C in children: Comparison with LDL direct measurement and Friedewald equation in the BLIP study. Nutr Metab Cardiovasc Dis. 2021;31(6):1911-1915.

25. Zafrir B, Saliba W, Flugelman MY. Comparison of novel equations for estimating low-density lipoprotein cholesterol in patients undergoing coronary angiography. J Atheroscler Thromb. 2020;27(12):1359-1373.

26. Sweeney T, Quispe R, Das T, Juraschek SP, Martin SS, Michos ED. The use of blood biomarkers in precision medicine for the Primary Prevention of atherosclerotic cardiovascular disease: A review. Expert Rev Precis Med Drug Dev. 2021;6(4):247-258. 


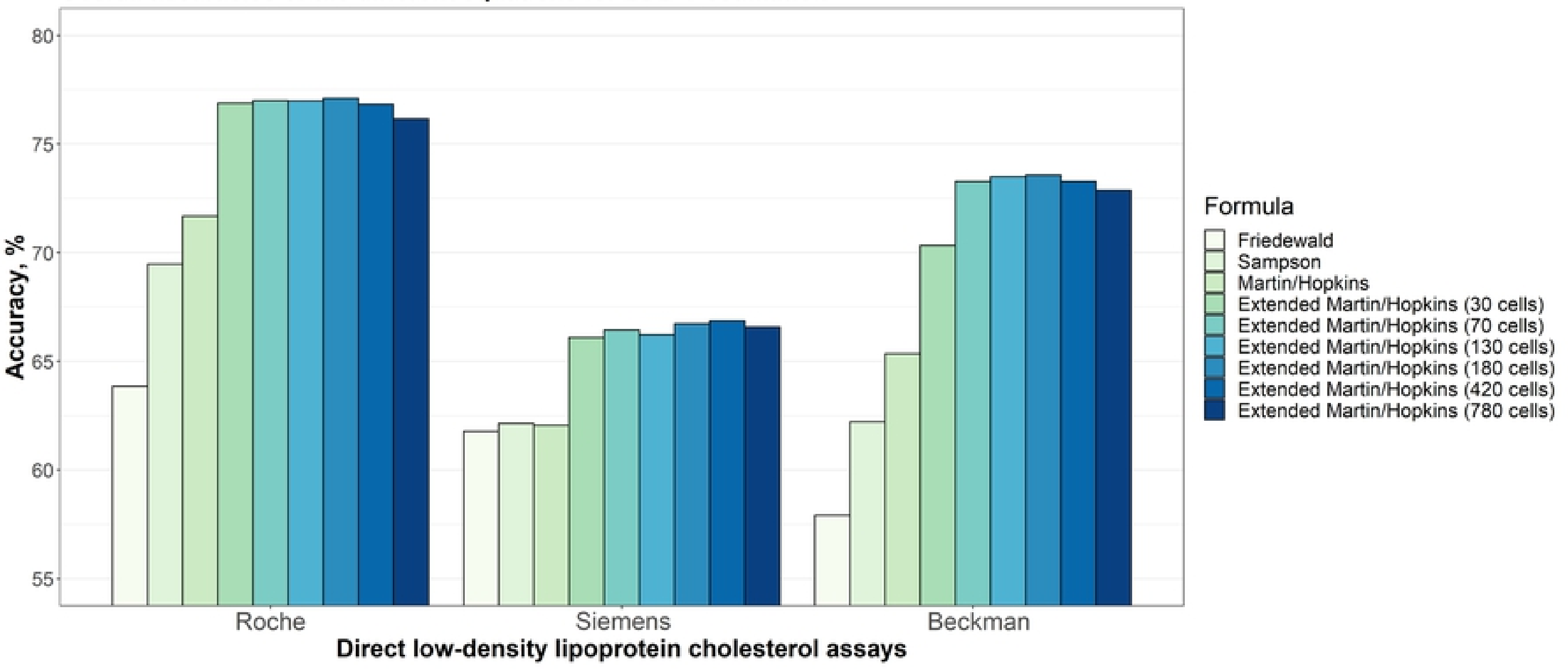

Figure 1 


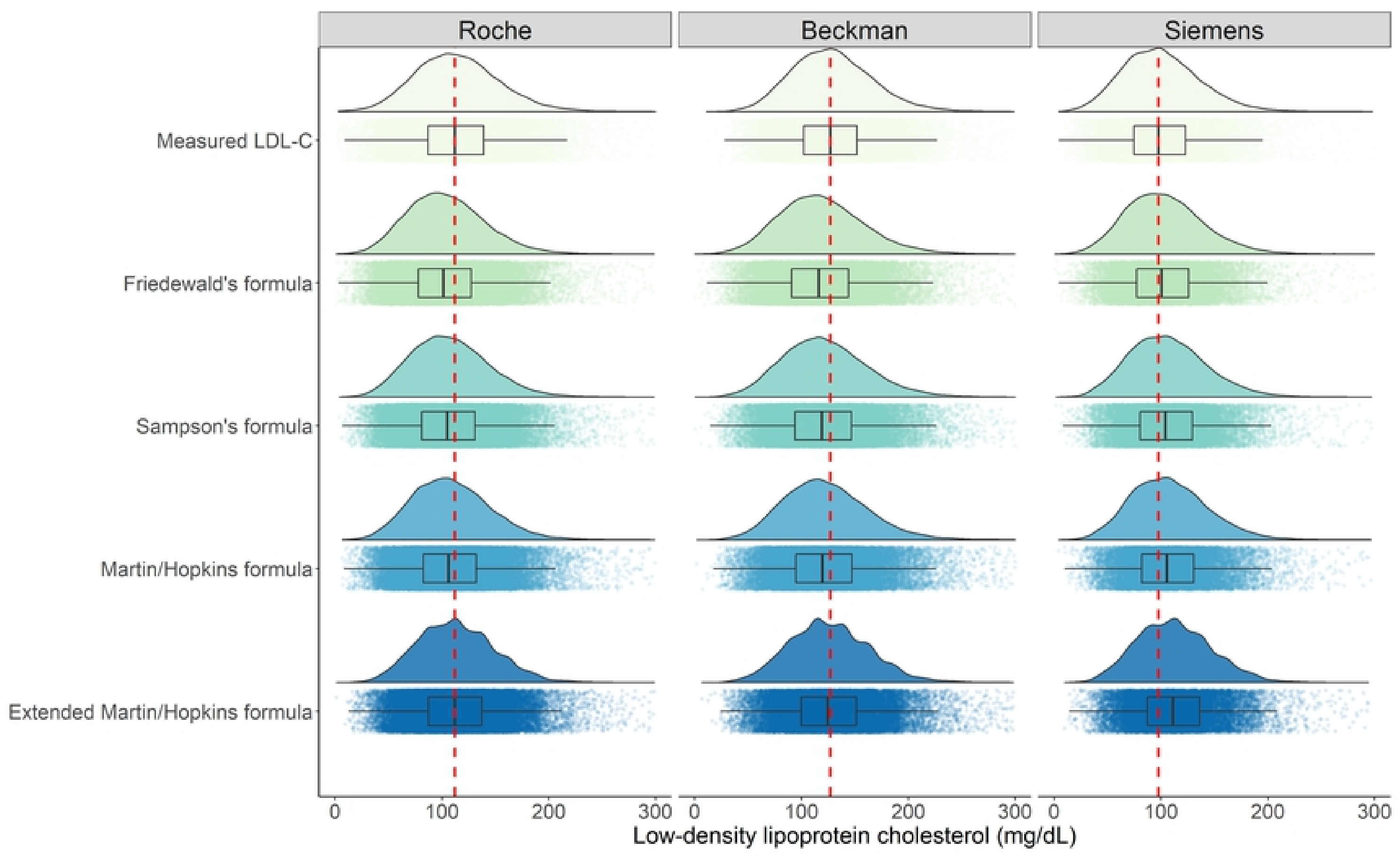

Figure 2 
Accuracies of the different equations for LDL-C estimation by LDL-C strata

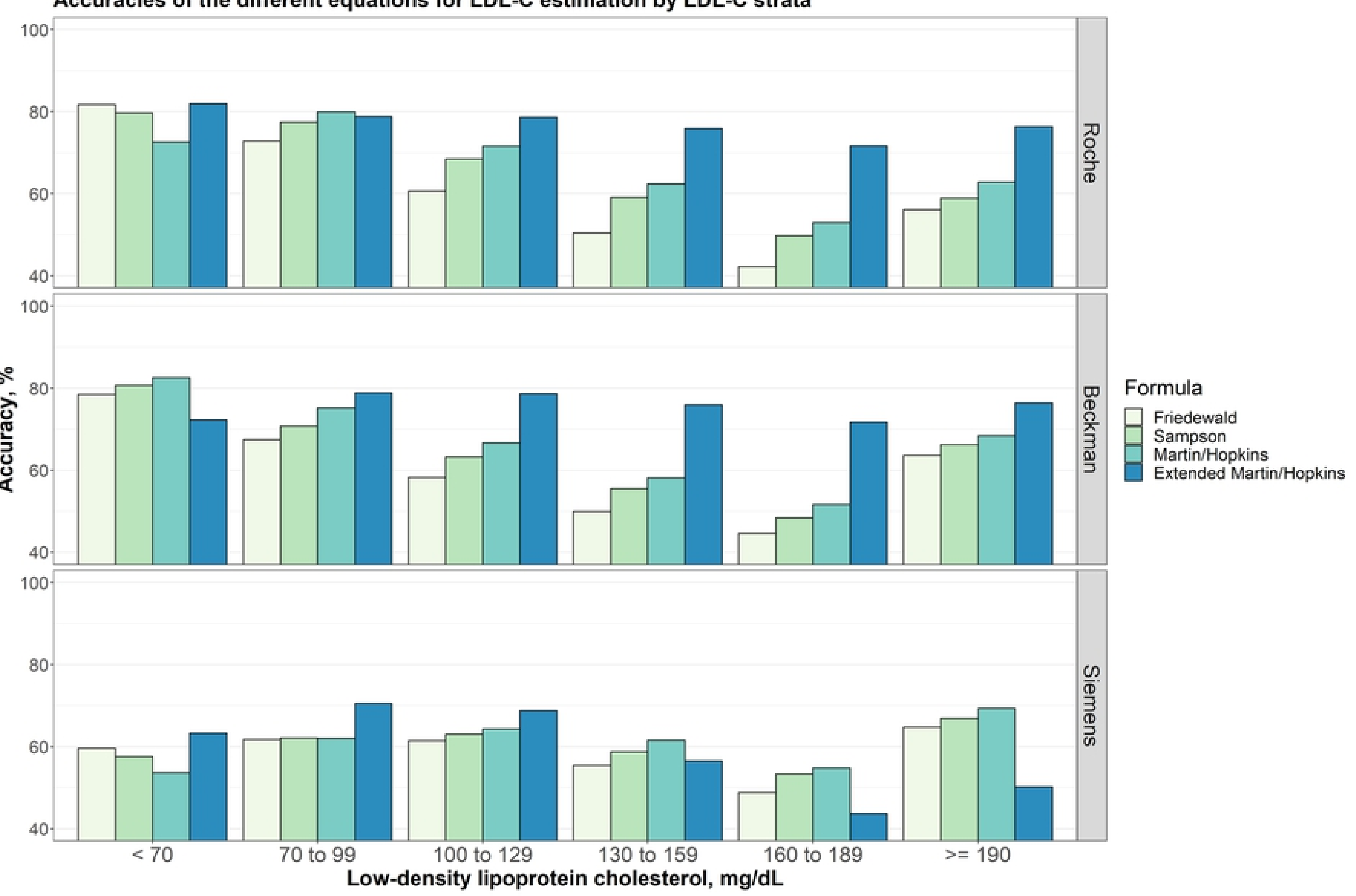

Figure 3 
Accuracies of the different equations for LDL-C estimation by triglycerides strata

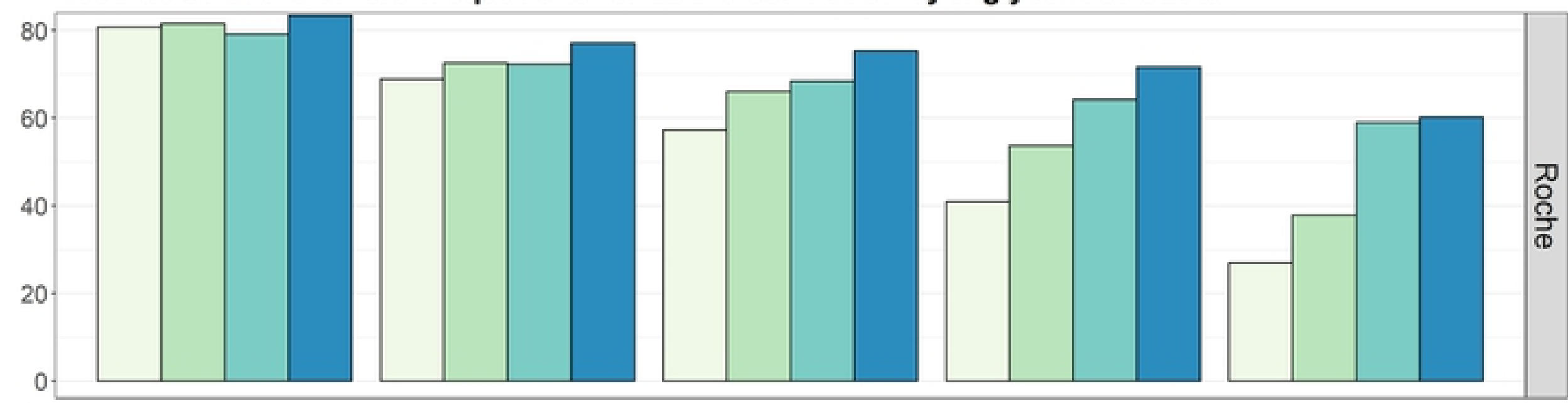

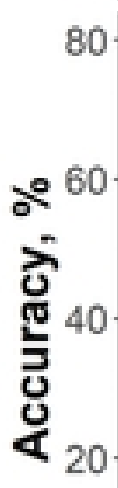

今.60
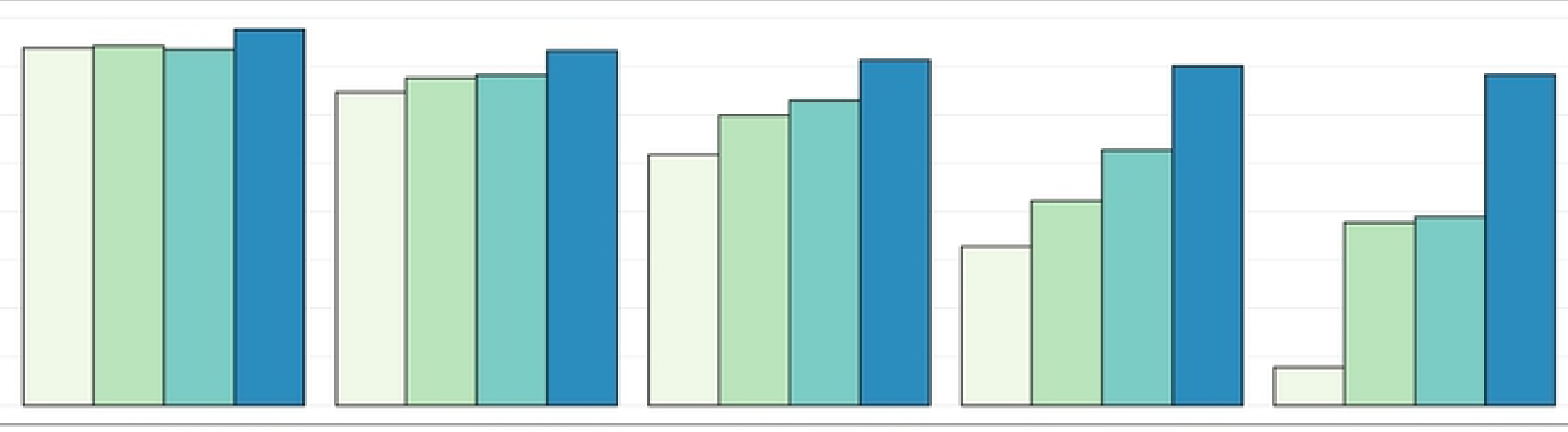

w Formula

웃 $\square$ Friedewald

슨 Sampson

@ $\square$ Martin/Hopkins

Extended Martin/Hopkins

80
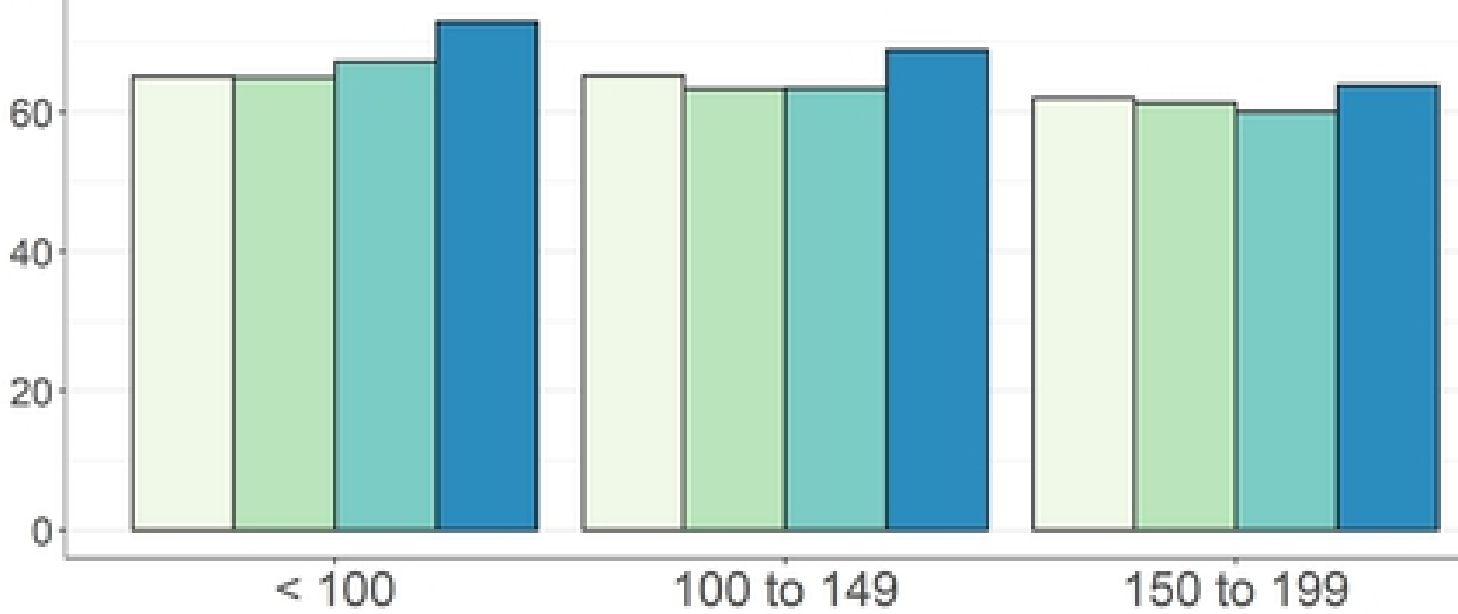

150 to 199

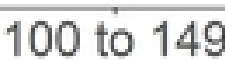

Trigylcerides, mg/dL

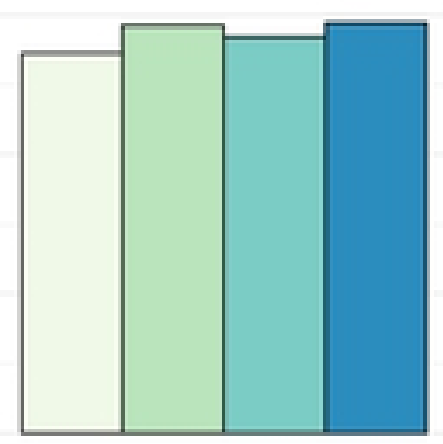

200 to 399

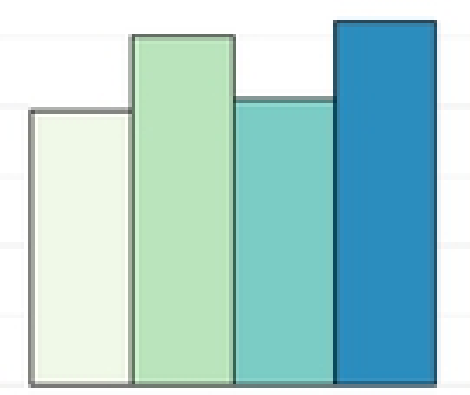

$\frac{\omega}{\frac{\omega}{D}}$

Figure 4 
Accuracies of the different equations for LDL-C estimation by non-HDL-C strata

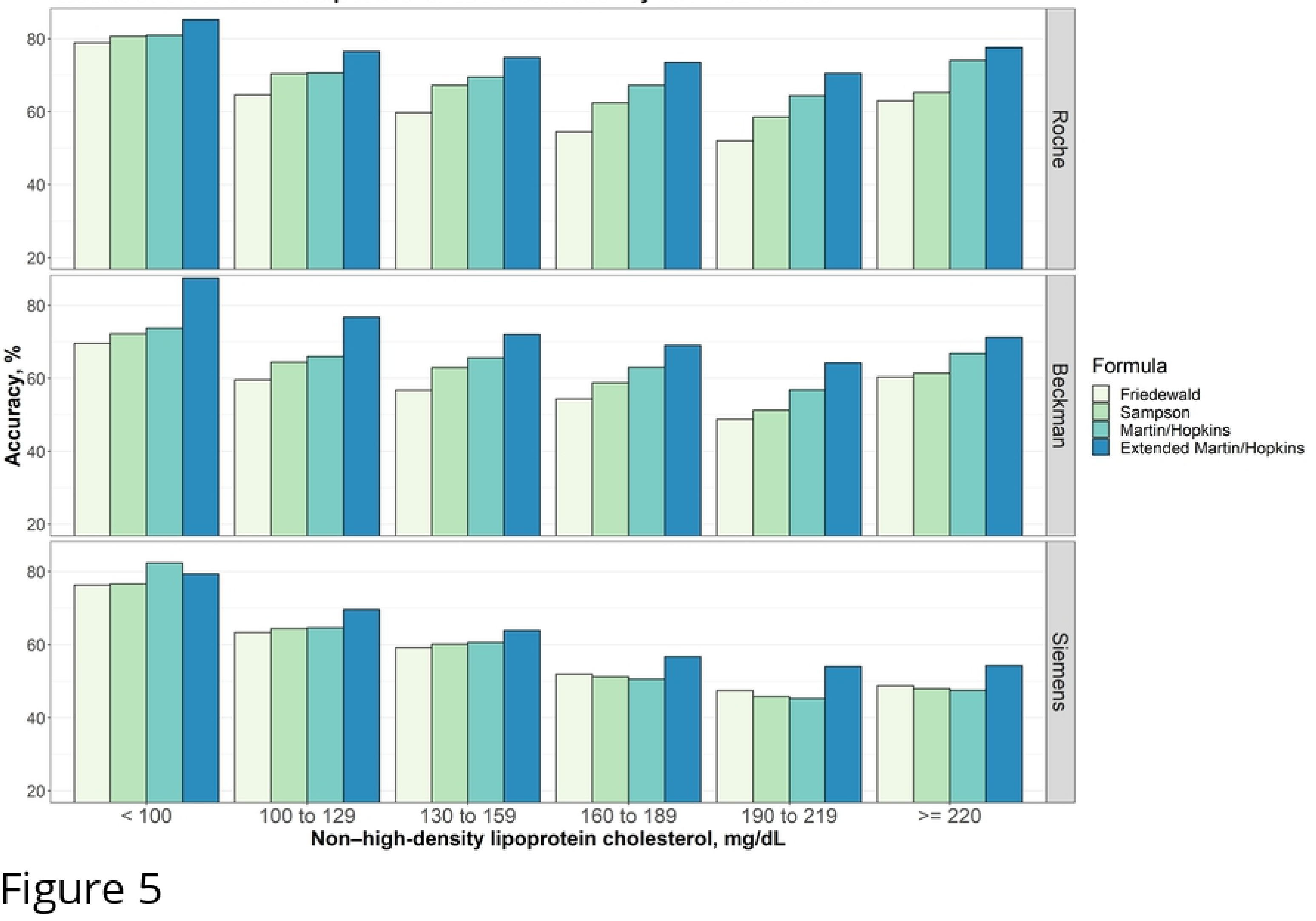


